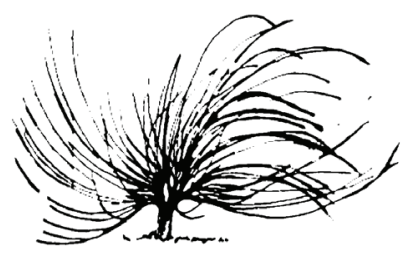

\title{
Efecto del programa "Un paso activo" en los hábitos de movimiento de estudiantes de primaria
}

\author{
Carlos Álvarez Bogantes ${ }^{1}$ \\ Universidad Nacional \\ Heredia, Costa Rica \\ ceab.03@gmail.com
}

\begin{abstract}
Resumen
El propósito de este artículo es evaluar el efecto de la propuesta Un paso activo en los hábitos de movimiento de estudiantes. Sujetos: 69 estudiantes de tercer grado de una escuela urbana pública del cantón central de San José, de los cuales 34 pertenecieron al grupo experimental (16 niños y 18 niñas) y 35 al grupo control (15 niños y 16 niñas), con edades promedio de 9.2 años, tomaron parte en el estudio. La intervención utilizó el acercamiento socio-constructivista de las lecciones de educación dado por el MEP en la organización de las lecciones de Educación Física. Diseño del estudio: Cuasiexperimental a través de 4 meses. Resultados: Los niños y niñas en el grupo experimental tuvieron un incremento significativo en el conocimiento, y en el tiempo reportado en actividad física a través del reporte dirigido $(<.05)$. Se presentó un efecto positivo en el cambio de actitud hacia la actividad física de las mujeres del grupo experimental.
\end{abstract}

\section{(c) (1) $\Theta \Theta$}

Recibido: 17 de mayo de 2017-Aprobado: 21 de mayo de 2018

http://dx.doi.org/10.15359/rep.13-1.3

1 Promotor de la Salud Fisica, Doctor en Educación, UNED 
Conclusiones: La estrategia pedagógica dada por el MEP, mejoró el tiempo dedicado a la actividad física y el estado de cambio de los participantes en el grupo experimental, especialmente en las niñas.

Palabras clave: Intervención, cambio conducta, actitud, actividad física.

\begin{abstract}
The purpose of this article was to evaluate the effect of the program "Un Paso Activo" on students' physical activity of elementary students. Subjects: 69 third-grade students from a public urban school in the central canton of San José, of which 34 belonged to the experimental group (16 boys and 18 girls) and 35 to the control group (15 boys and 16 girls), with average ages Of 9.2 years, took part in the study. The intervention was led by the project coordinator and the institution's physical education teacher, who provided the experimental group with a socio-constructivist approach to the lessons of Education given by the Ministry of Education in organizing the lessons of Physical Education, using the Active Step program as a methodological tool. Study Design: A quasiexperimental design was used over 4 months. Results: Boys and girls in the experimental group had a significant increase in knowledge, and in the time reported in physical activity through the directed report $(<.05)$. Conclusions: The pedagogical strategy given by MEP improved the time spent on physical activity and the state of change of participants in the experimental group, especially in girls. This study proved to be practical and showed that it could be easily incorporated into the educational context.
\end{abstract}

Keywords: Intervention, behavior change, attitude, physical activity 


\section{Introducción}

$\mathrm{L}$

os beneficios físicos, mentales y sociales de la actividad física en la niñez son ampliamente reconocidos (Demetrious y Höner, 2012; Janssen y LeBlanc, 2010). Recomendaciones internacionales para la niñez sobre la actividad física mencionan que deben realizar por lo menos 60 minutos diarias con una intensidad de moderada a vigorosa (WHO, 2010). Actualmente la población infantil muestra mayores niveles de sedentarismo, lo que la expone a una situación de riesgo a desarrollar sobrepeso y obesidad, así como enfermedades cardiovasculares o la diabetes (Álvarez, 2016; Hynynen et al, 2015). Cuando se habla de que en la etapa infantil se presentan sobrepeso y obesidad, uno de los puntos a revisar es la disminución de los niveles de actividad física, condición que podría trascender hasta la adolescencia, etapa en la cual se vuelve más evidente, lo que hace que se requieran estrategias de intervención efectivas y eficaces durante la infancia para contribuir a una niñez más activa y saludable (Janz, Burns y Levy, 2005).

A nivel mundial se han realizado intervenciones con el objetivo de aumentar la actividad física en el entorno escolar (Hynynen et al, 2015; Salmon et al., 2007). Estos estudios han mostrado una gran eficiencia al utilizar el entorno de la clase de educación física para influir en la disminución de factores de riesgo de enfermedades degenerativas, y en la disminución de los niveles de sedentarismo (Kahn et al, 2002; Ward et al, 2006); sin embargo, es difícil encontrar intervenciones que se hayan ejecutado en las cuales la prioridad sea la enseñanza de destrezas físicas, motoras y conductuales, herramientas fundamentales para moverse tanto en centros educativos como en el contexto del hogar.

En el marco de la prevención en salud de la niñez con sobrepeso y obesidad, se ha aceptado que el acercamiento más apropiado a la hora de desarrollar intervenciones para disminuir los niveles de sedentarismo es el modelo que se ejecuta en el contexto educativo, esto debido al hecho de que se considera a la totalidad de estudiantes en riesgo a tener sobrepeso y obesidad (CDC, 2013). Adicionalmente, los programas e intervenciones en promoción de salud escolar y de intervención en general, en muchas ocasiones son específicos para poblaciones con sobrepeso u obesidad, situación que trae muchas dificultades, las cuales van desde que sean sostenibles en el tiempo, hasta el impacto en las 
etiquetas que se le dan a sus participantes, situaciones que ayudan a que el impacto se vuelva reducido y los niveles de efectividad cuestionables (Biddle et al, 2011; Demetriou et al, 2012).

En respuesta a la importancia de la escuela como centro de promoción y prevención del sobrepeso y la obesidad, surge la asignatura Educación Física, como ente coordinador de esos esfuerzos sostenibles de una niñez en movimiento en los centros educativos (Cox, Schofield y Kold, 2010; MEP, 2013; Salmon et al., 2007; Timperio et al., 2004).

El hecho de usar enfoques didácticos constructivistas en esta intervención responde al deseo de ofrecer datos que muestren que la propuesta de estructuración de las lecciones de Educación Física, en los cuatro momentos que se presenta en los nuevos programas, ofrece los espacios de aprendizaje necesarios para el desarrollo de estilos de vida activos en los niños y niñas, rompiendo con los esquemas tradicionales y reduccionistas que han prevalecido en el sistema educativo costarricense (Álvarez, 2016).

Los momentos de la clase de Educación Física (MEP, 2013), para la promoción de estilos en movimiento en los niños y niñas de esta intervención, promovieron un proceso de reflexión que reconoció que los niños y niñas se encuentran en diferentes niveles de aptitud de cambio (Prochaska y Di Clemente, 1983), que deben contemplarse en el aprendizaje de destrezas físicas, conductuales y de disfrute de lests mismas.

El propósito principal de este estudio fue el de evaluar el impacto del programa de la metodología constructivista: "Un paso activo, en los patrones de movimiento de niños de primaria de tercer grado en las variables de actitud de cambio y en los niveles de la actividad física". Este artículo presenta los resultados de la intervención.

\section{Métodos}

\section{Participantes}

Se utilizó una muestra por conveniencia de 69 estudiantes de tercer grado de una escuela urbana pública del cantón central de San José, de los cuales 34 pertenecieron al grupo experimental (16 niños y 18 niñas) y 35 al grupo control (15 niños y 16 niñas). 


\section{Diseño e intervención}

La intervención con "Un Paso Activo", utilizó un diseño cuaexperimental con una duración de 14 semanas, con el objeto de incrementar la actividad física diaria del estudiantado a través del mejoramiento de las destrezas básicas y de la adquisición de destrezas conductuales y cognitivas desarrolladas en el modelo transteorético (Ward et al., 2007), en el entorno de la clase de Educación Física, con una metodología constructivista (MEP, 2013). La intervención consistió en utilizar la estrategia didáctica de 4 pasos utilizada en los nuevos programas de Educación Física. La intervención consistió en los siguientes momentos: en los primeros 5 o 10 minutos de la lección se hizo un recordatorio de los conceptos teóricos y prácticos abarcados en las lecciones previas, seguido del calentamiento dirigido por alguien del grupo. Una vez terminado el calentamiento, cada docente dirigió actividades físicas que enfatizaron el concepto teórico de la semana y el aprendizaje de destrezas físicas. Una vez finaliza esta sección, docente, junto con el estudiantado, realizaron una sesión de retroalimentación y movimiento exploratorio, donde los sujetos participantes reflexionaron sobre posibles formas de darle solución a la problemática vista en la clase teórica y práctica. Se finalizó con la distribución de las actividades extra clase o con la toma de decisiones en cuanto a las metas que cada quien se propondría en el incremento de estilo de vida activo y saludable.

\section{Variables y su medición}

Los niños y niñas del grupo control recibieron el acercamiento metodológico tradicional que prevalece en el sistema educativo costarricense, y estudiantes del grupo experimental recibieron el acercamiento constructivista de los nuevos programas de Educación Física, fueron evaluados y reevaluados con el recordatorio del día previo de actividad física (Weston et al., 1997). Este recordatorio está diseñado para obtener información acerca del tipo y nivel de la actividad física de participantes, requiere que se recuerden las actividades dominantes realizadas en el día previo en bloques de 30 minutos, reportando por cada actividad el nivel de intensidad percibida en una escala que contempla los rangos de sentado, parado, caminando o corriendo. Este recordatorio del día previo se considera una herramienta apropiada para 
determinar la cantidad de actividad que los niños y las niñas ejecutan durante el entorno escolar, especialmente si se supervisa y se realiza durante tres días típicos (Ward et al., 2007). El autorreporte presenta una correlación aceptable con el acelerómetro Caltrac $(r=0,39)$ (Kowalski, Crocker y Faulkner, 1997).

Adicionalmente, se utilizó la Escala de actitud de cambio desarrollada por Prochaska y DiClemnte (1983) validada en niñez por Haas y Nigg (2008), usando el cuestionario Godin's Leisure-Time Exercise Questionnaire $(1: \mathrm{F}=8.54, \mathrm{df}=4508, \mathrm{p}<.0125, \eta 2=.07$; sample 2: $\mathrm{F}=4.72, \mathrm{df}=4252, \mathrm{p}<.0125, \eta 2=.06)$. Este cuestionario utiliza un enfoque que explica cuándo y cómo cambian su comportamiento de actividad física a través del modelo de cambio de comportamiento y de la selección que hacen de alguno de los siguientes estadios, en relación con la actividad física: precontemplación, contemplación, preparación, actividad física o mantenimiento (Lox et el., 2006), lo que ofrece la posibilidad de entender la disposición mental de un individuo a comenzar la actividad física (Marcus et al., 2003).

\section{Métodos estadísticos}

En todas las variables medidas se les realizó una T de Student para determinar las condiciones iniciales de ambos grupos. Posterior a esto, se ejecutaron análisis de varianza (ANOVA $2 \times 2 \times 2$ ), para determinar el impacto de la metodología en la variable de actividad física, en los casos que se encontraron diferencias como resultado del tratamiento, se aplicó un test pos-hoc de múltiples comparaciones Tukey-Kramer. Con respecto a la actitud de cambio se realizó la prueba no-paramétrica de signos, para dar una respuesta más propicia al tipo de medida ordinal de esta prueba.

\section{Resultados}

El promedio de edad de los sujetos participantes fue de 9.2 (promedio $\pm \mathrm{SD}=9.2 \pm$ años). No hubo diferencias al inicio de la intervención entre grupos en cada una de las variables medidas.

Como se observa en la tabla 1 , el grupo experimental y control fueron clasificados, inicialmente, en los siguientes estadios de cambio de conducta hacia la actividad física: un $89 \%$ de las niñas estuvieron en 
la etapa de contemplación y un $46 \%$ de los niños estuvieron en la etapa de acción y un $50 \%$ en la etapa de mantenimiento.

\section{Tabla 1}

Porcentajes del pre y post tests en la variable de actitud de cambio de los participantes den el grupo experimental

\begin{tabular}{|l|l|l|}
\hline \multicolumn{1}{|c|}{ Proceso de cambio } & \multicolumn{1}{|c|}{ Hombres } & \multicolumn{1}{c|}{ Mujeres } \\
\hline & \multicolumn{1}{|c|}{ Pre Post } & \multicolumn{1}{c|}{ Pre Post } \\
\hline Precontemplación & \multicolumn{1}{|c|}{$3.1 \%$} \\
Contemplación & $3.4 \%$ & $89.5 \% 18.75 \%$ \\
Preparación & $7.14 \%$ & $6.2 \% 81.25 \%$ \\
Acción & $46.6 \% 21.42 \%$ & $3.1 \%$ \\
Mantenimiento & $50 \% 71.42 \%$ & \\
\hline Total & $100 \% 100 \%$ & $100 \% 100 \%$ \\
\hline
\end{tabular}

Con respecto a la variable de actitud de cambio, se encontró una interacción positiva entre las niñas del grupo experimental y control, en el pre test y post test. Esto demuestra un efecto positivo en el cambio de actitud hacia la actividad física de las mujeres del grupo experimental $(\mathrm{p}<.05)$, lo que indica que las niñas del grupo experimental tuvieron una modificación en la actitud de cambio hacia la actividad física como resultado de la intervención.

Tabla 2

Promedios y desviaciones estándar de la medición de actividad física del grupo experimental

\begin{tabular}{|c|c|c|}
\hline Variable & \multicolumn{1}{|c|}{ Mujeres } & \multicolumn{1}{c|}{ Hombres } \\
\hline & $\begin{array}{c}\text { Pre-test Post test } \\
\mathrm{x} \pm \text { SD } \mathrm{x} \pm \mathrm{SD}\end{array}$ & $\begin{array}{l}\text { Pre Post } \\
\mathrm{x} \pm \mathrm{SD} \mathrm{x} \pm \mathrm{SD}\end{array}$ \\
\hline Tiempo actividad física & 15.372 .4524 .533 .44 & 24.344 .0327 .783 .42 \\
\hline
\end{tabular}

En la tabla 2 se presentan los promedios y desviaciones estándar de la actividad física por día del grupo experimental. Al analizar la variable de incremento de tiempo dedicado a la actividad física, se encontró que hubo interacciones positivas entre el grupo experimental y control en el pre test y el post test, se notaron cambios mayores en las mujeres del grupo experimental en relación con los hombres del mismo grupo (<.05). Como resultado de la intervención, hubo incrementos 
significativos en el tiempo de actividad física del grupo experimental, donde fue mayor este incremento en las niñas. Es evidente que las niñas obtuvieron mayores cambios significativos en la variable en discusión, especialmente en el tiempo dedicado a la actividad física moderada, el cual fue un incremento de un $48 \% \%$ de mejoramiento.

\section{Discusión}

Desde una perspectiva de la promoción de la actividad física para la salud, este estudio ha proporcionado información útil, que muestra que usando la propuesta de organización del tiempo, amparado en una visión socio-constructivista de la lección de Educación Física como se presenta en los nuevos programas (MEP, 2013), se logra incrementar la actividad física de los niños y niñas, y causar un impacto evidente en la forma de valorar y de actuar con respecto al movimiento humano en sus vidas, con un progreso altamente significado en los estadios de cambio, especialmente, en las niñas.

Aunque los resultados pre-intervención de esta investigación no posibilitan mostrar una tendencia hacia la inactividad física de los sujetos participantes a través del tiempo de los sujetos participantes, si se podría mencionar que estos datos reflejan un alto grado de actitud sedentaria en el ámbito de las niñas, el cual coloca a esta población con una conducta tempranera de riesgo para el desarrollo de enfermedades cardio-vasculares, obesidad y diabetes (Thorp, Owen, Neuhaus, y Dunstan, 2011). Como resultado de la intervención, este riesgo disminuye, especialmente en las mujeres, por lo que el fomentar estilos de vida activos desde edades tempranas presenta valor preventivo benéfico (Wilmot et al., 2012).

Cuando observamos en los resultados del estudio, se nota el efecto positivo de la intervención utilizada en las variables de actitud de cambio y prácticas de actividad física, con respecto al grupo control. También, se evidenciaron los incrementos importantes en la actividad física del grupo de mujeres y en la actitud de cambio de ellas, hacia estilos de vida más activos, lo que es de suma importancia, por los niveles más altos de conductas sedentarias mostradas en las mujeres (Álvarez, 2016) en el contexto educativo. Esta situación, en particular, hace, del contexto educativo, un lugar prometedor para combatir la tendencia al incremento del sedentarismo entre las niñas, conforme avanzan en el 
sistema educativo; sin embargo, también llama la atención, a que las niñas requieren de diferentes estrategias, dependiendo de la edad, como lo expone Hynynen et al., (2015).

La necesidad particular de la promoción de la actividad física en las niñas y las adolescentes ha sido establecida, los estudios realizados para definir las estrategias en la promoción de la actividad física entre las niñas no muestran éxito en los resultados (Camacho et al., 2011).

Aunque los hallazgos de esta investigación ofrecen una panorámica alentadora con respecto al campo de la promoción de la salud física escolar, es claro que cuando los resultados son comparados con parámetros de la actividad física requerida por todo niño y niña para obtener beneficios para la salud (Hynynen et al, 2015; Jansen y LeBlanc, 2010), los alcances de esta intervención sugieren que todavía se requiere de aumentos, aunque estos sean mínimos, pero sobre todo valorar el tiempo de la intervención, con el fin de garantizar adherencia hacia conductas activas, en el tiempo. Una revisión sistemática de revisiones y metaanálisis sobre los efectos de las intervenciones demostró que las imás efectivas fueron las que generalmente duraron tres meses, y consideraron como idóneas las intervenciones de mayor duración (Dudley et al., 2011; Safron et al., 2001).

Pareciera que la intervención realizada en esta investigación fue más efectiva cuando se compara al tiempo utilizado en las intervenciones clásicas como la de "Conozca su cuerpo" (Walter et al., 1985) o la del proyecto Familia saludable (Nader et al., 1996), los cuales duraron entre dos meses a un año. Estos estudios reportaron pocos incrementos en la actividad física de sus participantes. En la intervención Saltando por su salud (Álvarez, 2016) se mostraron cambios significados en los niveles de actividad física en el contexto educativo y un incremento de la autoeficacia; sin embargo, esta fue una intervención multi-nivel donde intervinieron no solo las lecciones de educación fisca, sino también el contexto de escuela y las políticas hacia la actividad física escolar. En el estudio de Harrel y compañeros (1998), llamado "Salud cardiovascular de los niños", lógicamente se obtuvieron mayores resultados en el ámbito de actividad física, pero fue un estudio más intenso, que se basó en actividad física todos los días y clases magistrales durante un año.

Dudle et al. (2011) han reportado niveles altos de éxito al realizar intervenciones en la clase de educación física, incrementando la cantidad de actividad moderada ofrecida durante estos espacios. Sin 
embargo, estas intervenciones fueron realizadas durante todo la semana y no comprobaron que sus participantes mantuvieran la actividad fuera de la clase.

Los hallazgos de esta intervención se ven avalados por estudios clásicos que persisten hasta el presente, como CATCH (McKenzie et al., 1996) y SPARK (Sallis et al., 1997) que fueron guiados por la teoría del aprendizaje social (Bandura, 1977) y M-SPAN, que se basa en la teoría ecológica (Bronfenbrenner, 1979). La evidencia sugiere que las intervenciones desarrolladas en referencia a una teoría del cambio de comportamiento son más exitosas en el cambio de comportamiento que las que carecen de ese referente teórico (Lubans, Foster y Biddle, 2008; Michie y Abraham, 2004); sin embargo, los hallazgos de esta investigación sugieren que un acercamiento socio-constructivista aporta elementos novedosos dentro del contexto de clase, en la construcción de la alfabetización física que promueve el Ministerio de Educación.

Esta intervención partió del hecho de que cada niño o niña está en una etapa diferente de preparación para el cambio hacia estilos de vida activos, por lo que no se asumió que estuvieran listos para hacer un cambio de comportamiento inmediato o permanente. Mediante la identificación de la posición de un niño o niña en el proceso de cambio, se incentivó la intervención a la etapa de preparación del sujeto participante, lográndose cambios sustanciales en la etapa de cambio de los participantes del grupo experimental, especialmente en las niñas. Se puede explicar que el mayor nivel de avance de las niñas a través de los estadios de cambio se atribuye a que ellas presentaron niveles más bajos en la escala al inicio de la intervención, lo que permitió mayores niveles de avance hacia fases superiores, lo cual queda evidenciado con los mayores niveles de actividad física que se presentaron en el las niñas post test. Aunque las niñas no avanzaron a través de la escala de cambio en forma secuencial, el progreso dado refuerza la idea de ajustar las lecciones al estado en que se encuentra la mayoría del alumnado (Van Sluijs et al., 2004), con el fin de obtener mejores resultados. Evidentemente, el resultado más prometedor en el progreso de las niñas lleva a recomendar un desarrollo de la clase que contemple el uso de estrategias múltiples que impacten a cada participante (Gorely y Bruce, 2000; Lox et al., 2006).

En el caso del uso de la estrategia recomendada por el Ministerio de Educación (2013) en las clases de Educación Física, se decidió 
utilizar una intervención que no implicara inclusión de nuevos elementos en el currículo, por lo que lo que se realizó fue un reordenamiento de los contenidos en salud que el personal educador físico ya realizaba. El articular los conocimientos en salud permitió un refuerzo constante entre cada uno de los contenidos abarcados.

Esta intervención usó como conductor del proceso de intervención a un profesor de Educación Física; al respecto, diferentes revisiones han sugerido que cuando se capacita apropiadamente a la persona conductora del proceso de intervención, se garantiza la entrega de la propuesta en forma exacta y clara (Greaves et al., 2011, Peters, Kok, Ten Dam, Buijs, y Paulussen, 2009), lo cual establece que las intervenciones conducidas por el equipo investigador eran más eficaces que las proporcionadas por el personal de la escuela u otro tipo proveedor, esta situación podrían influir en los resultados finales y disminuir la reproducción de la vivencia.

\section{Conclusión}

Gran cantidad de conductas relacionadas con la salud son formadas en la niñez, lo que sugiere que la escuela primaria ofrece un punto crucial para intervenir. Se necesita identificar programas de intervención que no solo sean efectivos, sino que también respeten la idiosincrasia de los niños y niñas escolares de Costa Rica, que efectivamente mejoren las conductas en salud, especialmente las relacionadas con la actividad física, con el afán de reducir los factores de riesgo cardiovasculares. Este estudio es el primer estudio conocido que interviene a niños y niñas escolares con gran éxito a un costo ínfimo.

Este programa demostró que el usar la propuesta metodológica socio-constructivista de organización de las lecciones de Educación Física logró un incremento sustancial en relación con el tiempo dedicado a la actividad física, aunque, en esta última variable, no se lograron los valores recomendados para la salud. En la variable de cambio de conducta se alcanzaron cambios prometedores y exitosos en el uso del modelo utilizado, fundamentalmente en las niñas.

Se sugiere, sin embargo, para lograr resultados más prometedores en las variables estudiadas, trabajar estrategias más orientados a integrar las propuestas cognitivas y conductuales, de los diferentes niveles de actitud de cambio que se encuentran entre el estudiantado de primaria. 


\section{Referencias}

Álvarez, C. (2016). Efectividad del programa ecológico "Saltando por su salud" en la promoción de la actividad física y la auto-eficacia en niños y niñas escolares de tercer grado. Revista Ensayos pedagógicos, 11(1), 147-169.

Bandura, A. (1977). Self-efficacy: The exercise of control. New York: Freeman.

Biddle, S. J. H., O'Connell, S., y Braithwaite, R. E. (2011). Sedentary behaviour interventions in young people: A meta-analysis. British Journal of Sports Medicine, 45(11), 937-942.

Bronfenbrenner, U. (1979). The Ecology of Human Development: Experiments by Nature and Design. Harvard University Press,Cambridge, MA.

Camacho-Min, M., Nicole M., LaVoi Daheia J, y Barr-Anderson. (2011). Interventions to promote physical activity among young and adolescent girls: a systematic review. Health Education Research, 26(6), 1025-49.

Center for Disease Control and Prevention [CDC]. (2013). Make a Difference at Your School. Recuperado de http://digitalcommons. hsc.unt.edu/cgi

Cox, M., Schofield, G., y Kolt, G. S. (2010). Responsibility for children's physical activity: parental, child, and teacher perspectives. J. Sci. Med. Sport, 13, 46-52.

Demetrious, Y., y Höner, O. (2012). Physical activity interventions in the school setting: A systematic review. Psychology of Sport and Exercise, 13(2), 186-196.

Dudley, D., Okely, A., Pearson, P., y Cotton,W., (2011). A systematic review of the effectiveness of physical education and school sport interventions targeting physical activity, movement skills and enjoyment of physical activity. Eur. Phys. Educ. Rev, 17, 353-378.

Greaves, C., Sheppard, K., Abraham, C., Hardeman, W., Roden, M., Evans, P., y Schwarz, P. (2011). Systematic review of reviews of intervention components associated with increased effectiveness in dietary and physical activity interventions. Bio-Med, 11, 2-12.

Gorely, T, y Bruce, D. (2000). A 6-month investigation of exercise adoption from the contemplation stage of the transtheoretical model. Psychology of Sport and Exercise, 1(2), 89-101. 
Haas, S y Nigg C. (2008). Construct validation of the stages of change with strenuous, moderate, and mild physical activity and sedentary behaviour among children. Journal of Science and Medicine in Sport, 12, 86-591.

Harrell, S., Gansky, S., McMurray, R., Frauman, A., y Bradley, C. (1998). School based interventions improve heart health in children with multiple cardiovascular disease risk factors. Pediatrics, 102, 371-380.

Hynynena, S.,M. M. van Stralenb, F. F. Sniehottac, V. Araújo-Soaresc, W. Hardemand, M. J. M. Chinapawe, T. Vasankarif y. Hankonena. (2015). A systematic review of school based interventions targeting physical activity and sedentary behaviour among older Adolescents. International Review Of Sport And Exercise Psychology, 9(1), 22-44. http://dx.doi.org/10.1080/1750984X.2015.1081706

Janssen, I., y LeBlanc, A. G. (2010). Review systematic review of the health benefits of physical activity and fitness in school-aged children and youth. International Journal of Behavioral Nutrition and Physical Activity, 7(40), 1-16.

Janz K. F., Burns T. L., y Levy, S. M. (2005). Tracking of Activity and Sedentary Behaviors in Childhood: The Iowa Bone Development Study. Am J Prev Med, 29(3), 171-178.

Kahn, E. B., Ramsey, L. T., Brownson, R. C., Heath, G. W., Howze, E. H., Powell, K. E., Stone, E. J., Rajab, M. W., y Corso, P. (2002). The effectiveness of interventions to increase physical activity: a systematic review. Am J Prev Med, 22, 73-107.

Kowalski, K., Crocker, P., y Faulkner, R. (1997). Validation of the Physical Activity Questionnaire for Older Children. Pediatric Exercise Science, 174-186.

Lox, C., Martín, K. y Petruzzello, S. (2006). The Psychology of Exercise. Arizona: HHP.

Lubans, D. R., Foster, C., y Biddle, S. J. H. (2008). A reviewofmediators of behavior in interventions to promote physical activity among children and adolescents. Prev. Med, 47, 463-470. 20.

McKenzie, T. L., Nader, P. R., Strikmiller, P. K., Yang, M. (1996). School physical education: Effectof the child and adolescent trial for cardiovascular health. Prev.Med, 25, 423-431.

Marcus, B. y Forsyth, L.(2003). Motivating People to be Physical Active. Champaign, IL: Human Kinetics. 
MEP. (2013). Programas de Estudio de Educación Física. San José, Costa Rica: Autor.

Michie, S., y Abraham, C. (2004). Interventions to change health behaviours: evidencebased or evidence-inspired? Psychol. Health, 19, 29-49.

Nader, P., Sellers, D., Johnson, C., Perry ,C., Stone, E., Cook, K., Bebchuk, J, and Luepker, R. (1996). The effect of adult participation in a school-based family intervention to improve Children's diet and physical activity: the Child and Adolescent Trial for Cardiovascular Health. Revista end linea Pub-Med, 25(4),455-64. Recuperado de https://www.ncbi.nlm.nih.gov/pubmed/8818068

Peters, L. W., Kok, G., Ten Dam, G., Buijs, G. J., y Paulussen, T. (2009). Effective elements of school health promotion across behavioral domains: A systematic review of reviews. BMC Public Health, 9(1), 182-196.

Prochaska, J. y DiClemente, C. (1983). The Transtheoretical Model. Recuperado de http://ocw.unican.es/ciencias-de-la-salud/promocion-de-la-salud/material-de-clase/ tema-3.-el-modelo-transteorico/skinless_view

Safron, D, Schulenberg, J y Bachman, J (2001). Part-time work and hurried adolescence: The links among work intensity, social activities, health behaviors, and substance use. Journal of Health and Social Behavior, 42, 425-449. 10.2307.3090188. [PubMed].

Sallis, J., McKenzie, T. L., Alcaraz, J., Kolody, B., Faucette, N., y Hovell, M. (1997). The effects of a 2-year physical education program (SPARK) on physical activity and fitness in in elementary school students. Sports, Play and Active Recreation for Kids. Am J Public Health, 87(8), 1328-34.

Salmon, J., Booth, M. L., Phongsavan, P., Murphy, N., y Timperio, A. (2007). Promoting Physical Activity Participation among Children and Adolescents. Epidemiol Rev, 29, 144-159. 23.

Salmon, J., y Timperio, A. (2007). Prevalence, trends and environmental influences on child and youth physical activity. Med Sport Sci, 50, 183-199. 15.

Thorp, A., Owen, N., Neuhaus, M, y Dunstan, D. (2011). Sedentary behaviors and subsequent health outcomes in adults a systematic review of longitudinal studies. American Journal of Preventive Medicine, 41(2), 207-215. 
Timperio, A., Salmon, J., y Ball, K. (2004). Evidence-based strategies to promote physical activity among children, adolescents and young adults: review and update. Journal of Science and Medicine in Sport, 7(Suppl 1), 20-29.

Van Sluijs, E., Van Poppel, M., Stalman, W., y Van Mechelen, W. (2004). Feasibility and acceptability of a physical activity promotion program in general practice. Family Practice, 21, 429-436.

Walter, H., Hofman, A., Connelly, P., Barrett, L, y Kost, K. (1985). Primary prevention of chronic disease in childhood: changes in risk factors after one year of intervention. Am J Epidemiol, 122, 772-81.

Ward, D., Saunders, R., y Pate, R. (2007). Physical Activity Interventions in Children and Adolescents. Champaign, Il: Human Kinetics Publishers.

Weston, A., Petosa, R., y Pate, R. (1997). Validation of an instrument for measurement for physical activity in youth. Medicine and Science in Sport and Exercise, 29, 138-143.

WHO. (2010). Recomendaciones mundiales sobre actividad fisica. Ginebra, Suiza: OMS.

Wilmot, E. G., Edwardson., C. L., Achana, F. A., Davies, M. J., Gorely, T., Gray, L. J, y Biddle, S. J. H. (2012). Sedentary time in adults and the association with diabetes, cardiovascular disease and death: Systematic review and meta-analysis. Diabetologia, $55,2895-2905$. 